\title{
Attivazione dei recettori della Vitamina D nell'insufficienza renale cronica
}

\author{
Marina Di Luca, Silvio Di Stante, Hirissanti Kulurianu, Flavia Manenti, Mauro Marani, \\ Mauro Martello
}

\author{
U.O. Nefrologia e Dialisi, A.O. Ospedali Riuniti Marche Nord, Presidio San Salvatore, Pesaro
}

\begin{abstract}
VDR ACTIVATION IN CKD
Abstract. Disturbances of mineral metabolism occurs in virtually all patients during the progression of chronic kidney disease (CKD), because the decreasing functional renal mass and the reduction in renal 1a-hydroxylase activity lead to defective renal production of calcitriol at very early CKD stages. Beyond the effect of parathyroid hormone suppression, an increasing body of experimental data suggests that vitamin $D$ exerts pleiotropic effects, which have been associated with an improvement of cardiovascular disease and mortality rate in observational studies. Recent data from randomized controlled studies showed that paracalcitol treatment in CKD patients also results in a significant reduction of albuminuria, which is a major risk factor for cardiorenal syndrome outcome. However, the current K/DOQI and KDIGO recommendations limit the administration of VDR activation agents for the sole treatment of hyperparathyroidism. The role of different vitamin D metabolites and their analogues, administered in CKD patients, will be discussed in this review.
\end{abstract}

Key words: Vitamin D, Chronic kidney disease, Secondary hyperparathyoidism, VDR activators, Paricalcitol

Conflict of interest: None.

Ricevuto: 24 Aprile 2013; Accettato: 29 Aprile 2013

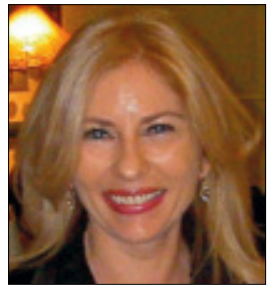

Marina Di Luca

\section{Introduzione}

Nei pazienti affetti da insufficienza renale cronica (IRC), i livelli sierici di 1.25 -idrossivitamina $\mathrm{D}\left(1.25(\mathrm{OH})_{2} \mathrm{D}_{3}\right)$ e di 25 -idrossivitamina $\mathrm{D}\left(25(\mathrm{OH}) \mathrm{D}_{3}\right)$ si riducono fin dagli stadi iniziali e la prevalenza di deficit significativi aumenta al ridursi del filtrato glomerulare (VFG), prima che si manifestino anomalie nella concentrazione ematica di calcio e fosforo e prima che si evidenzi un aumento dei livelli di paratormone (PTH); il deficit di Vitamina D endogena è la causa principale di iperparatiroidismo secondario (IPTS) (1). La ridotta attivazione del recettore per la Vitamina D (VDR) è determinata sia da ridotti livelli di $25(\mathrm{OH}) \mathrm{D}_{3}$ che di $1.25(\mathrm{OH})_{2} \mathrm{D}_{3}$. Le cause del deficit di $25(\mathrm{OH}) \mathrm{D}_{3}$ sono rappresentate dalla riduzione della quota circolante filtrata a livello glomerulare e dall'alterazione del meccanismo di endocitosi nelle cellule tubulari prossimali per ridotta espressione della megalina. Il declino progressivo di $1.25(\mathrm{OH})_{2} \mathrm{D}_{3}$ è determinato dalla ridotta attività dell'enzima 1-alfa idrossilasi renale, a causa della riduzione della massa nefronica funzionante, e dall'inibizione dell'attività enzimatica da parte di tossine uremiche, frammenti di PTH e aumentata produzione di FGF-23 (2). I composti attivatori dei VDR (VDRa) disponibili per l'uso clinico possono essere suddivisi in due principali gruppi: i pro-farmaci, cioè quelli che necessitano di un'attivazione enzimatica (in posizione $25 \mathrm{e} / \mathrm{o}$ in posizione 1), e i farmaci attivi, in grado di legarsi direttamente al VDR (Tab. I). Nell'ultimo decennio, sono stati immessi sul mercato tre nuovi composti di sintesi: paracalcitolo, 22-oxacalcitriolo (maxacalcitolo) e doxercalciferolo, che, grazie a modifiche nella catena laterale del calcitriolo, presentano migliori caratteristiche di efficacia e maggiore tollerabilità, in termini di minore incidenza di ipercalcemia e iperfosforemia; il paracalcitolo e il 22-oxacalcitriolo sono definiti anche "VDRa Selettivi" (VDRa s), in relazione al reclutamento e all'attivazione di gruppi di geni differenti rispetto al capostipite calcitriolo (Tab. II). L'attivazione dei VDR determina: (a) effetti endocrini (classici) su osso, intestino, rene e paratiroidi: aumento dell'assorbimento intestinale del calcio, stimolazione del riassorbimento del calcio a livello del tubulo distale renale, stimolazione degli osteoblasti e dell'osteoclastogenesi e soppressione di secrezione, sintesi e crescita delle cellule paratiroidee; (b) effetti sistemici grazie all'ubiquitarietà del VDR in quasi tutti i tessuti, con meccanismo d'azione autocrino e paracrino per la presenza dell'attività enzimatica 1-alfa idrossilasi espressa a livello extrarenale (3). Una riduzione dell'attivazione dei VDR, oltre a determinare effetti 
negativi sul metabolismo osseo e sullo sviluppo dell'IPTS, si associa a numerosi outcome clinici sfavorevoli: aumento dell'infiammazione, disregolazione del sistema immunitario, aumento della proteinuria, attivazione del sistema renina-angiotensina, fibrosi miocardica e, da ultimo, possibile aumento della mortalità (4).

\section{Effetti Clinici dell'attivazione dei VDR}

La valutazione dei diversi VDRa deve prendere in esame non solo gli effetti sul controllo dell'iperparatiroidismo e sull'incidenza di ipercalcemia e iperfosforemia ma anche i possibili effetti sul sistema cardiovascolare, sul danno renale e, in particolare, sulla proteinuria e, da ultimo, l'influenza sulla mortalità generale e per cause cardiovascolari.

TABELLA I - NOMENCLATURA DELLA VITAMINA D

\begin{tabular}{lll}
\hline Vitamina D nativa & $\begin{array}{l}\text { Ergocalciferolo } \\
\text { Colecalciferolo }\end{array}$ & $\mathrm{D}_{2}$ \\
25-OH Vitamina D & Calcifediolo $\left(25(\mathrm{OH}) \mathrm{D}_{3}\right)$ & $\mathrm{D}_{3}$ \\
& & $\mathrm{D}_{3}$ \\
VDRA & Alfacalcidolo $\left(1(\mathrm{OH}) \mathrm{D}_{3}\right)$ & $\mathrm{D}_{3}$ proormone sintetico $^{\mathrm{a}}$ \\
& Doxercalciferolo $\left(1(\mathrm{OH}) \mathrm{D}_{2}\right)$ & $\mathrm{D}_{3}$ proormone sintetico \\
& Calcitriolo $\left(1.25(\mathrm{OH})_{2} \mathrm{D}_{3}\right)$ & $\mathrm{D}_{3}$ ormone naturale \\
& Paracalcitolo $\left(19 \mathrm{nor}, 1.25(\mathrm{OH})_{2} \mathrm{D}_{2}\right)$ & $\mathrm{D}_{2}$ analogo sintetico \\
& Maxacalcitolo $\left(22 \mathrm{oxa}, 1.25(\mathrm{OH})_{2} \mathrm{D}_{3}\right)$ & $\mathrm{D}_{3}$ analogo sintetico \\
\hline
\end{tabular}

VDRA: attivatori dei recettori della Vitamina D; 25-OH Vitamina D: 25-idrossivitamina D.

aProormone che richiede la 25 -idrossilazione nel fegato per diventare un ormone attivo.

TABELLA II - EFFETTI CLINICI E BIOLOGICI E PROBLEMATICHE DI UTILIZZO DELLA VITAMINA D E DEGLI ANALOGHI NELL'INSUFFICIENZA RENALE CRONICA

\begin{tabular}{|c|c|c|}
\hline Tipi di Vitamina D & Effetti clinici e biologici & Problematiche di utilizzo \\
\hline Alfacalcidolo & $\begin{array}{l}\text { Efficace nell'aumentare i livelli di } 25-\mathrm{D} \text { e } 1.25 \text {-D in } \\
\text { pazienti con insufficienza renale cronica iniziale o con } \\
\text { normale funzione renale }\end{array}$ & $\begin{array}{l}\text { Richiede l'attivazione nel rene per generare la forma attiva } \\
1.25-\mathrm{D}\end{array}$ \\
\hline Ergocalciferolo, colecalciferolo & $\begin{array}{l}\text { Efficace nell'aumentare i livelli di } 25-\mathrm{D} \text { e } 1.25-\mathrm{D} \text { in } \\
\text { pazienti con insufficienza renale cronica iniziale o con } \\
\text { normale funzione renale }\end{array}$ & $\begin{array}{l}\text { Richiede l'attivazione nel rene per generare la forma attiva } \\
\text { 1.25-D } \\
\text { Fornisce solo una parziale soppressione del paratormone } \\
\text { negli stadi più avanzati dell'insufficienza renale }\end{array}$ \\
\hline Calcitriolo & $\begin{array}{l}\text { Agonista biologicamente attivo del recettore della } \\
\text { Vitamina D } \\
\text { Sopprime in maniera efficace l'iperparatiroidismo } \\
\text { secondario } \\
\text { Riduce l'anormale turnover osseo }\end{array}$ & $\begin{array}{l}\text { L'utilizzo ad alte dosi può provocare ipercalcemia, } \\
\text { iperfosforemia e ipercalciuria }\end{array}$ \\
\hline Doxercalciferolo & $\begin{array}{l}\text { Sopprime l'iperparatiroidismo secondario come il } \\
\text { calcitriolo o meglio } \\
\text { Evidenziata riduzione dei valori di osteocalcina } \\
\text { e di fosfatasi alcalina ossea }\end{array}$ & $\begin{array}{l}\text { Richiede l'attivazione nel fegato per generare } \\
1.25-\mathrm{D} \\
\text { Provoca significativi aumenti della fosforemia, } \\
\text { con la conseguente necessità di un maggiore utilizzo di } \\
\text { chelanti del fosforo }\end{array}$ \\
\hline Paracalcitolo & $\begin{array}{l}\text { Agonista biologicamente attivo del recettore della } \\
\text { Vitamina D } \\
\text { Sopprime in maniera efficace l'iperparatiroidismo } \\
\text { secondario } \\
\text { Evidenziata riduzione dei valori di osteocalcina } \\
\text { e di fosfatasi alcalina ossea }\end{array}$ & $\begin{array}{l}\text { Lieve aumento di calcemia, fosforemia e prodotto calcio x } \\
\text { fosforo, richiedente un monitoraggio } \\
\text { di calcemia e fosforemia }\end{array}$ \\
\hline
\end{tabular}




\section{Soppressione del PTH}

Nel 2009 è stata pubblicata una Review della Cochrane Libra$r y$ sull'utilizzo di VDRa nei pazienti con IRC in terapia conservativa; sono stati inclusi nell'analisi 16 studi randomizzati controllati per un totale di 894 casi, trattati con ogni forma di Vitamina D, e sono stati analizzati i risultati in termini di outcome biochimici e "patient centered" outcome: mortalità, inizio del trattamento dialitico e istomorfometria ossea (5). La terapia con calcitriolo ha determinato in 5 studi randomizzati controllati la significativa riduzione del PTH e il miglioramento dei parametri istomorfometrici ossei, ma con un incremento della calcemia e, in uno studio, anche della fosforemia. La terapia con alfacalcidolo ha determinato, in 3 studi randomizzati controllati, riduzione del PTH e miglioramento dei parametri istomorfometrici ossei, con un incremento significativo della calcemia. In letteratura è riportato un solo studio randomizzato controllato con utilizzo di doxercalciferolo vs placebo: il PTH si riduceva del $46 \%$ dopo 24 settimane di trattamento, senza variazioni significative di calcemia e fosforemia rispetto al gruppo di controllo. Si registravano, peraltro, un aumento della percentuale di pazienti a cui veniva prescritto un chelante dei fosfati e un aumento significativo della calciuria nel gruppo trattato (6). Sull'efficacia e sulla tollerabilità dell'uso del paracalcitolo nell'IRC due recenti metanalisi di 6 studi randomizzati controllati dimostrano che i pazienti trattati avevano una significativa riduzione del PTH durante il periodo di osservazione (7). Nello studio di Coyne, il paracalcitolo somministrato a 113 pazienti con IRC agli stadi 3 e 4 vs 110 in placebo determinava, nell'arco di 6 mesi, riduzioni del PTH ( $\geq 30 \%$ ) nel $91 \%$ dei casi rispetto al 13\% osservato nel gruppo placebo, senza significative variazioni di calcemia e fosforemia, con un'immodificata escrezione renale di calcio e fosforo, e senza un incremento della percentuale di pazienti che venivano trattati con chelanti dei fosfati (8). Il paracalcitolo è anche risultato più efficace nella soppressione del PTH rispetto alla somministrazione di Vitamina $\mathrm{D}_{2}$ (ergocalciferolo) in 80 pazienti con IPTS in IRC agli stadi 3 e 4 e deficit di $25(\mathrm{OH}) \mathrm{D}_{3}(<30 \mathrm{ng} / \mathrm{mL})$, in assenza di episodi di ipercalcemia e iperfosforemia (9). Sanchez, in uno studio osservazionale su una popolazione di 92 pazienti, dei quali il 55.4\% con IRC allo stadio 3 e il $44.5 \%$ con IRC allo stadio 4 , otteneva dopo 6 mesi di trattamento una riduzione dei livelli di PTH $>30 \%$ (mediana da 163 a $81 \mathrm{pg} / \mathrm{mL}$ ) nel 70.6\% dei casi, con un'incidenza di ipercalcemia nel 5.4\% dei casi, in assenza di iperfosforemia (10).

\section{Protezione cardiovascolare}

L'attivazione dei VDR determina su cuore e vasi vari effetti, importanti per il mantenimento di una struttura e di una funzione dell'apparato cardiovascolare normali: modificazione delle resistenze a livello della parete arteriosa, riduzione della trombogenicità, stimolo della rigenerazione endoteliale e riduzione della sintesi e della secrezione di renina (11). In uno studio su ratti con gene nullo per 1-alfa idrossilasi, si sviluppava ipertrofia ventricolare sinistra (IVS), che veniva attenuata dalla somministrazione di $1.25(\mathrm{OH})_{2} \mathrm{D}_{3}(12)$. In un altro modello di ratto uremico, è stato dimostrato che il paracalcitolo stimola l'espressione dei VDR nel miocardio e sopprime la progressione dell'ipertrofia ventricolare sinistra e la fibrosi miocardica e perivascolare; pertanto, l'attivazione dei VDR potrebbe esercitare un impatto clinico positivo sulla cardiomiopatia uremica, complicanza, questa, comune nell'IRC e caratterizzata da fibrosi cardiaca, ipertrofia cardiaca e disfunzione diastolica (13). Nel 1999, è stato pubblicato uno studio randomizzato controllato su 30 pazienti in emodialisi cronica con IVS e IPTS (PTH $>450 \mathrm{pg} / \mathrm{mL}$ ), che aveva l'obiettivo di valutare l'effetto del calcitriolo sull'IVS: 15 pazienti venivano trattati con calcitriolo alla dose di $2 \mathrm{mcg}$ e.v. a fine dialisi per due volte/settimana e venivano osservati per 15 settimane vs placebo; la massa ventricolare sinistra si riduceva significativamente nel gruppo trattato con calcitriolo, da 178 a $155 \mathrm{gr} / \mathrm{m}^{2}$, mentre rimaneva quasi invariata nel gruppo di controllo. Parallelamente, nel gruppo trattato, si osservava una riduzione significativa del PTH e dei livelli plasmatici di renina (14). Lo studio PRIMO è un trial randomizzato controllato multicentrico che si è svolto dal 2008 al 2010 allo scopo di valutare se la terapia con paracalcitolo alla dose di $2 \mathrm{mcg} /$ die riduce l'IVS in pazienti con IRC agli stadi $3 \mathrm{~b} / 4$, con ipertrofia ventricolare sinistra lieve-moderata e normale funzione sistolica e PTH compreso tra 50 e $300 \mathrm{pg} / \mathrm{mL}$. Sono stati inclusi nello studio 220 pazienti (110 per braccio) ed è stata effettuata una valutazione dell'indice di massa ventricolare sinistra con risonanza magnetica nucleare (RMN) ed ecocardiografia, dopo 24 e 48 settimane di trattamento con paracalcitolo alla dose di $2 \mathrm{mcg} /$ die. Gli end point secondari comprendevano indici ecocardiografici di funzione diastolica, biomarker cardiaci e tasso di ospedalizzazioni e mortalità. Al basale non vi erano differenze nei valori di pressione arteriosa, uso di diuretici, inibitori del sistema renina angiotensina e agenti stimolanti l'eritropoiesi. I livelli di PTH si riducevano in misura $>30 \%$ nell' $85.7 \%$ dei pazienti trattati entro 4 settimane dall'inizio della terapia e si mantenevano stabili per la durata del follow-up, mentre i livelli di calcemia e fosforemia aumentavano significativamente nel gruppo trattato con un'incidenza di ipercalcemia del $22.6 \%$ vs placebo $0.9 \%(\mathrm{P}<001)$. A 48 settimane, 1'end point primario non veniva raggiunto: il paracalcitolo non modificava nel gruppo trattato gli indici di massa ventricolare sinistra, né gli indici di funzione diastolica, mentre vi era una differenza significativa nel tasso di ospedalizzazioni per cause cardiovascolari tra il gruppo trattato vs placebo (15). Una possibile ipotesi del mancato effetto sulla riduzione dell'IVS potrebbe risiedere nel periodo di follow-up relativamente breve (48 settimane) e nella selezione di pazienti con un grado "lieve" di IVS al basale (23.7 g/m² alla RMN) (16). In un'analisi post-hoc dello studio PRIMO viene evidenziato che il paracalcitolo riduceva in maniera statisticamente significativa l'indice di volume atriale sinistro (LAVi) $\left(-2.79 \mathrm{~mL} / \mathrm{m}^{2}\right.$ [95\% CI da $\left.\left.-4.00 \mathrm{a}-1.59 \mathrm{~mL} / \mathrm{m}^{2}\right]\right)$ rispetto al gruppo placebo $(-0.70 \mathrm{~mL} /$ $\mathrm{m}^{2}$ [95\% CI da -1.93 a $\left.\left.0.53 \mathrm{~mL} / \mathrm{m}^{2}\right], \mathrm{P}=0.02\right)$; questo dato è clinicamente rilevante nei pazienti con IVS, poiché è riconosciuta l'associazione del LAVi con gli eventi cardiovascola- 
ri, in particolare con l'insufficienza cardiaca congestizia, le ospedalizzazioni e la mortalità; è stato, inoltre, evidenziato un ridotto incremento dei livelli di BNP nel gruppo trattato $(+10.8 \%$ in paracalcitolo vs $+21.3 \%$ in placebo, $\mathrm{P}=0.02)$ (17). Il miglioramento di questi parametri può essere interpretato come un indice indiretto di miglioramento della funzione diastolica del ventricolo sinistro (18).

\section{Protezione renale}

Nell'ultimo decennio, numerose evidenze sperimentali hanno documentato gli effetti renoprotettivi dell'attivazione dei VDR, che sono determinati dall'inibizione diretta del sistema renina angiotensina (RAS) intrarenale, dell'infiammazione, attraverso l'inibizione di NF-kB e altri fattori di trascrizione, e della neo-fibrogenesi e dall'induzione dell'attività di Klotho e che si traducono in vivo in una riduzione della proteinuria. $(19,20)$. È ben noto il ruolo della proteinuria quale fattore di rischio indipendente sulla progressione del danno renale e sul rischio cardiovascolare e quale target terapeutico per rallentare la progressione della malattia renale cronica (21). Da qui, l'interesse della comunità scientifica per il possibile ruolo di VDRa sulla renoprotezione (Tab. III). La prima segnalazione circa l'effetto antiproteinurico del paracalcitolo deriva da un'analisi secondaria condotta da Agarwal nel 2005 dei dati di 3 studi randomizzati su un totale di 220 pazienti; l'Autore ha valutato i 118 pazienti che mostravano proteinuria al basale (determinata con metodica semiquantitativa), rilevando che il $51 \%$ dei pazienti del gruppo randomizzato a paracalcitolo $(\mathrm{n}=57)$ dopo 6 mesi presentava una riduzione della proteinuria rispetto al $25 \%$ dei pazienti randomizzati a placebo $(\mathrm{n}=61)$ $(\mathrm{P}=0.004)$, indipendentemente dalla concomitante terapia con anti-RAS (22). A questo è seguito un trial pilota in 24 pazienti con IRC, trattati con inibitori del RAS, randomizzati a ricevere paracalcitolo $1 \mathrm{mcg}$ o $2 \mathrm{mcg}$ vs placebo per la durata di 1 mese; tra gli end point primari, oltre all'azione antiproteinurica, vi era anche la valutazione della funzione endoteliale e dei marker infiammatori (PCR); l'albuminuria aumentava del $35 \%$ nel gruppo placebo, mentre diminuiva del $48 \%$ nel gruppo trattato con $1 \mathrm{mcg}$ e del $46 \%$ nel gruppo trattato con 2 mcg, indipendentemente da variazioni del VFG o della pressione arteriosa; la riduzione significativa della PCR (dal 20\% al 30\% nei trattati) suggeriva anche un effetto antinfiammatorio del paracalcitolo (23). Nel 2009 veniva pubblicato lo studio condotto da Fishbane, controllato in doppio cieco, in cui sono stati randomizzati 61 pazienti con filtrato glomerulare compreso tra 15 e $90 \mathrm{~mL} / \mathrm{min}$ e proteinuria maggiore di 400 $\mathrm{mg} /$ die a ricevere paracalcitolo alla dose di $1 \mathrm{mcg}$ vs placebo: nel gruppo trattato si otteneva una riduzione della proteinuria (determinata sul rapporto proteinuria/creatininuria) del 17.6\% $(\mathrm{P}<0.05)$ vs $+2.9 \%$ nel gruppo placebo (24). Nel 2010 sono stati pubblicati i risultati dello studio VITAL ("Selective vitamin D receptor activation with paricalcitol for reduction of albuminuria in patients with type 2 diabetes), condotto su 281 pazienti con diabete tipo 2 e albuminuria compresa tra 11 e $339 \mathrm{mg} / \mathrm{mmol}$ (misurata come rapporto albuminuria/ creatininuria-UACR) e un VFG di $15-90 \mathrm{~mL} / \mathrm{min}$ per $1.73 \mathrm{~m}^{2}$, in trattamento con inibitori del RAS, randomizzati a ricevere per 24 settimane paracalcitolo alla dose di 1 o $2 \mathrm{mcg} / \mathrm{die}$. La riduzione di UACR è risultata di $-16 \%$ nel gruppo trattato con paracalcitolo vs $-3 \%$ nel gruppo placebo $(\mathrm{P}=0.071)$; la variazione di UACR seguiva una relazione dose-risposta, infatti nel gruppo che assumeva $1 \mathrm{mcg} /$ die la differenza vs placebo è stata di $-11 \%(95 \% \mathrm{CI}$ da $-27 \mathrm{a}-8)(\mathrm{p}=0.23)$, mentre, nel gruppo che assumeva $2 \mathrm{mcg} /$ die, la riduzione di UACR è stata di $-18 \%(95 \%$ CI da -32 a 0$)(\mathrm{P}=0.053)$. Un dato interessante

TABELLA III - STUDI RANDOMIZZATI SUGLI EFFETTI DEL TRATTAMENTO CON PARACALCITOLO NELL'IRC

\begin{tabular}{|c|c|c|c|c|c|c|c|}
\hline & $\begin{array}{l}\text { Tipo di } \\
\text { studio }\end{array}$ & $\begin{array}{l}\text { N. } \\
\text { Pazienti }\end{array}$ & Popolazione & Disegno dello studio & Follow-up & End point primario & Risultati \\
\hline Coyne et al. (9) & $3 \mathrm{RCT}$ & 220 & - IRC stadi 3-4 & $\begin{array}{c}\text { Randomizzazione } \\
\text { 1:1 PCT* vs placebo }\end{array}$ & 24 settimane & $\begin{array}{l}\text { Almeno due riduzioni } \\
\text { consecutive dei livelli } \\
\text { di iPTH } \geq 30 \%\end{array}$ & $\begin{array}{l}\text { End point conseguito nel } \\
91 \% \text { dei pazienti PCT vs } \\
13 \% \text { dei pazienti placebo } \\
\qquad(\mathrm{P}<0.001)\end{array}$ \\
\hline Agarwal et al. (22) & $3 \mathrm{RCT}$ & 220 & - IRC stadi 3-4 & & & $\begin{array}{l}\text { Riduzione della } \\
\text { proteinuria al dipstick }\end{array}$ & $\begin{array}{l}\text { End point conseguito nel } \\
51 \% \text { dei pazienti } \mathrm{PCT} \text { vs } \\
25 \% \text { dei pazienti placebo } \\
\qquad(\mathrm{P}<0.004)\end{array}$ \\
\hline Fishbane et al. (24) & $\mathrm{RCT}$ & 61 & - IRC stadi 2-4 & $\begin{array}{l}\text { Randomizzazione } \\
\text { 1:1 PCT } 1 \mathrm{mcg} / \text { die } \\
\text { vs placebo }\end{array}$ & 24 settimane & $\begin{array}{l}\text { Valutazione variazioni } \\
\text { nell'uPCR medio }\end{array}$ & $\begin{array}{c}\text { uPCR medio: } \\
\text {-17.6\% nei pazienti PCT vs } \\
+2.9 \text { nei pazienti placebo }\end{array}$ \\
\hline VITAL (25) & $\mathrm{RCT}$ & 281 & $\begin{array}{l}\text { - IRC stadi 3-4 } \\
\text { - DM tipo } 2 \\
\text { - UACR: } 100- \\
3000 \mathrm{mg} / \text { die }\end{array}$ & $\begin{array}{l}\text { Randomizzazione } \\
\text { 1:1 PCT } 1 \mathrm{mcg} \\
\text { vs PCT } 2 \mathrm{mcg} \\
\text { vs placebo }\end{array}$ & $\begin{array}{c}24 \text { settimane } \\
\text { (dati preliminari) }\end{array}$ & $\begin{array}{l}\text { Riduzione dell'UACR al } \\
\text { temine del follow-up }\end{array}$ & $\begin{array}{l}\text { Riduzione UACR }>15 \% \\
\text { nei pazienti trattati con } \\
\text { PCT vs pazienti placebo } \\
\text { (dati preliminari) }\end{array}$ \\
\hline
\end{tabular}

$\mathrm{PCT}=$ paracalcitolo; $\mathrm{uPCR}=$ rapporto proteinuria/creatininuria; $\mathrm{UACR}=$ rapporto albuminuria/creatininuria; $\mathrm{RCT}=$ randomized controlled trial. *PCT $1 \mathrm{mcg} /$ die (o $2 \mathrm{mcg}$ a giorni alterni) per valori di $\mathrm{iPTH} \leq 500 \mathrm{pg} / \mathrm{mL}$ e PCT $2 \mathrm{mcg} / \mathrm{die}$ (o $4 \mathrm{mcg}$ a giorni alterni) per valori di iPTH $>500 \mathrm{pg} / \mathrm{mL}$. 
è rappresentato dalla maggiore riduzione di UACR nel sottogruppo di pazienti trattati con $2 \mathrm{mcg} /$ die di paracalcitolo e con un'elevata escrezione di sodio urinario $(>178 \mathrm{mmol} /$ die $)$. Durante l'osservazione, il valore di VFG subiva una riduzione significativa dalla $4^{\mathrm{a}}$ settimana di trattamento nel gruppo trattato con $2 \mathrm{mcg} /$ die $(\mathrm{P}=0.0548$ vs placebo $)$ e rimaneva stabile con un range tra -3 e $-5 \mathrm{~mL} / \mathrm{min}$ per $1.73 \mathrm{~m}^{2}(\mathrm{P}=0.001$ vs placebo $)$; inoltre, nel gruppo trattato con $2 \mathrm{mcg} /$ die, l'analisi per misure ripetute dei valori di PA durante le 24 settimane di osservazione mostrava una riduzione statisticamente significativa (range da -3 a $-9 \mathrm{mmHg} ; \mathrm{P}=0.033$ vs placebo), ma l'analisi di regressione multipla escludeva che l'effetto antiproteinurico del paracalcitolo dipendesse dalle variazioni di VFG e pressione arteriosa. L'incidenza di eventi avversi non era statisticamente significativa nei 2 gruppi, con particolare riguardo a episodi di ipercalcemia. Gli Autori concludono che, pur non essendo stato raggiunto l'end point primario della riduzione significativa dell'albuminuria nel gruppo combinato (paracalcitolo a $1 \mathrm{mcg}$ e $2 \mathrm{mcg} /$ die), il paracalcitolo potrebbe rappresentare un'efficace terapia addizionale per la renoprotezione in pazienti con diabete tipo 2 e nefropatia, specialmente nei casi di non compliance alla dieta iposodica e di conseguente resistenza all'effetto antiproteinurico degli inibitori del RAS (25). Questa opzione è stata valutata nella pratica clinica in uno studio prospettico osservazionale multicentrico condotto in 48 pazienti con VFG $38 \pm 18 \mathrm{~mL} / \mathrm{min} / 1.73 \mathrm{~m}^{2}$, una proteinuria di $1.23(1.00-1.51) \mathrm{g} / 24 \mathrm{~h}$, in terapia con farmaci anti-RAS alla dose massima tollerata prima dell'inizio dello studio, e una sodiuria di $161 \pm 63 \mathrm{mEq} /$ die, posti in terapia con paracalcitolo alla dose di $1 \mathrm{mcg} /$ die, per un follow-up di 6 mesi. I valori di PTH si riducevano da 206 (mediana 102-270) pg/mL al basale a 90 (51-188) $\mathrm{pg} / \mathrm{mL}$ al $6^{\circ}$ mese e risalivano a 111 (74-184) $\mathrm{pg} / \mathrm{mL} 3$ mesi dopo la sospensione del paracalcitolo $(\mathrm{P}<0.05$ vs basale). La variazione mediana della proteinuria era, dopo 6 mesi, del 32\% (IQR 11-52) e il 38\% dei pazienti mostrava una remissione della proteinuria $(<0.5 \mathrm{~g} / 24 \mathrm{~h})$, senza che vi fosse una correlazione significativa con i valori di PA sistolica $(\mathrm{r}=0.146, \mathrm{P}=0.324)$.L'effetto antiproteinurico era maggiore per gradi inferiori di $\operatorname{IRC}(\mathrm{P}=0.004)$, nei diabetici $(\mathrm{P}=0.022)$ $\mathrm{e}$ in presenza di un'elevata introduzione di sodio con la dieta $(\mathrm{P}=0.005)$; i valori della proteinuria mediana tornavano ai livelli basali dopo 3 mesi dalla sospensione del paracalcitolo, da $1.23(1.00-1.51)$ a $1.32(1.02-1.71) \mathrm{gr} / 24 \mathrm{~h}(\mathrm{p}=0.501)(26)$. I dati della letteratura sull'azione renoprotettiva di altri VDRa sono più limitati rispetto al paracalcitolo. Liu et al., in un trial randomizzato controllato in 50 pazienti con normofunzione renale e glomerulonefrite $\operatorname{IgA}$, già in terapia massimale con inibitori del RAS, hanno descritto che l'impiego di calcitriolo a basse dosi $(0.5 \mathrm{mcg} / 2$ volte a settimana) determina una significativa riduzione della proteinuria di $-19 \%$ (da 1.60 a $1.30 \mathrm{~g} / 24 \mathrm{~h}$; 95\% CI, 42\%-4\%) nel gruppo trattato $(\mathrm{P}=-0.03)$ vs un aumento del $21 \%$ (da 1.29 a 1.58 g/24 h; 95\% CI, 9\%-52\%) nel gruppo placebo (27). L'effetto antiproteinurico di alfacalcidolo è stato, invece, descritto solo in uno studio osservazionale retrospettivo in 59 pazienti con IRC agli stadi 4 e 5 con macroalbuminuria (albuminuria $1.96 \pm 1.97 \mathrm{~g} / 24 \mathrm{~h}$ ), che iniziavano una terapia per IPTS; la riduzione dell'albuminuria è stata del $12 \%(\mathrm{P}<0.05)$ alla dose mediana di alfacalcidolo di $2.4 \mu \mathrm{g} /$ sett (range $0.75-7$ $\mu \mathrm{g} / \mathrm{sett}$ ), per un periodo di osservazione medio di 201 giorni tra la raccolta urinaria al basale e post trattamento; peraltro, $\mathrm{i}$ livelli di VFG si riducevano in maniera statisticamente significativa da 14.7 a $13.0 \mathrm{~mL} / \mathrm{min}, \mathrm{P}>0.001$, anche se gli Autori non riportano differenze statisticamente significative della velocità di riduzione della funzione renale residua (RRF) rispetto al gruppo di pazienti $\left(\mathrm{n}^{\circ} 52\right)$ che non assumevano alfacalcidolo (28).

\section{Mortalità}

La possibilità che la terapia con VDRa influenzi la mortalità dei pazienti con IRC è stata esplorata solo in studi di tipo osservazionale. Shoben ha valutato l'associazione tra uso di calcitriolo orale con la mortalità e l'ingresso in dialisi in una popolazione di 1418 pazienti con IRC (VFG $29 \mathrm{~mL} / \mathrm{min} / 1.73 \mathrm{~m}^{2}$ ); sono stati selezionati 429 pazienti che iniziavano la terapia con calcitriolo: $48 \%$ allo stadio 3 e $52 \%$ allo stadio 4 con IPTS e assenza di ipercalcemia vs 989 soggetti comparabili per età e VFG, non trattati. Durante un follow-up mediano di 1.9 anni, 408 (29\%) pazienti erano deceduti e 217 (16\%) iniziavano la dialisi. L'uso di calcitriolo si associava a un rischio di mortalità inferiore del $26 \%(95 \%$ IC $5-42 \% ; \mathrm{P}=0.016)$ e il beneficio persisteva dopo un aggiustamento per caratteristiche demografiche, comorbidità, funzione renale, farmaci e valori basali di PTH, nonostante un aumentato rischio di ipercalcemia e iperfosforemia nei soggetti trattati (29). Simili risultati sono stati descritti da Kovesdy in 520 soggetti maschi (età $69.8+/-$ 10.3 anni) con VFG di $30.8+/-11.3 \mathrm{~mL} / \mathrm{min}$; 258 pazienti ricevevano calcitriolo alla dose di $0.25 / 0.5 \mathrm{mcg} /$ die per una durata di 2.1 anni (range 0.06-6.0): il rischio di mortalità era inferiore del $35 \%$ rispetto ai non trattati; anche in questa analisi il rischio non si modificava nel modello statistico aggiustato per possibili fattori confondenti (30). Questo tipo di studi ha, però, delle rilevanti limitazioni: in primis, il disegno di tipo osservazionale retrospettivo permette unicamente il rilievo di un'associazione tra due variabili e non permette un'inferenza di causalità; inoltre, pur applicando modelli statistici aggiustati, non si possono escludere bias di selezione dei pazienti che ricevono il trattamento rispetto ai non trattati.

\section{Conclusioni}

Le evidenze a disposizione dimostrano che, nei pazienti con IRC non in dialisi, la correzione dell'iperparatiroidismo secondario può essere ottenuta in maniera efficace con tutte le tipologie di VDRa, ma, tra gli analoghi di sintesi, il paracalcitolo presenta un profilo di sicurezza e tollerabilità maggiore rispetto ai composti di prima generazione, in termini di una ridotta incidenza di ipercalcemia e iperfosforemia. Circa l'effetto antiproteinurico della Vitamina D nelle sue varie forme, il paracalcitolo è risultato il farmaco più interessante per i risultati ottenuti in 4 trial randomizzati controllati, anche se per brevi follow-up, mentre i dati attualmente disponibili non sono ancora sufficienti per raccomandare l'uso di VDRa ai fini della protezione cardiovascolare e della riduzione della mortalità. Ulteriori studi saranno necessari per stabilire se, nei pazienti 
con IRC, vi sia l'indicazione a iniziare una terapia con VDRa allo scopo di ripristinare il "deficit ormonale" di $1.25(\mathrm{OH})_{2} \mathrm{D}_{3}$, indipendentemente dalla necessità di controllare le alterazioni del metabolismo minerale.

\section{Riassunto}

I pazienti affetti da IRC presentano fin dai primi stadi della malattia una ridotta attivazione del recettore per la Vitamina D (VDR), determinata da ridotti livelli sia di 1.25-idrossivitamina $\mathrm{D}\left(1.25(\mathrm{OH})_{2} \mathrm{D}_{3}\right)$ che di 25 -idrossivitamina $\mathrm{D}$ $\left(25(\mathrm{OH}) \mathrm{D}_{3}\right)$, con il conseguente sviluppo di anomalie del metabolismo osseo e minerale. La Vitamina D e i suoi metaboliti di sintesi, oltre a controllare l'iperparatiroidismo secondario (IPTS), esercitano numerosi effetti pleiotropici in diversi sistemi cellulari e, in particolare, sembrano giocare un ruolo fondamentale nella salute del sistema cardiovascolare e nella riduzione della mortalità, come suggerito da studi osservazionali. È stato di recente dimostrato che il paracalcitolo orale nell'IRC non solo riduce il paratormo- ne, ma è anche associato a un effetto antiproteinurico significativo, e ciò appare di particolare interesse in quanto la proteinuria è un fattore di rischio renale e cardiovascolare. I dati attuali non sono, tuttavia, sufficienti per raccomandare l'uso degli agenti attivatori dei VDR (VDRa) oltre al trattamento delle alterazioni del metabolismo minerale legate all'iperparatiroidismo secondario.

Parole chiave: Vitamina D, Insufficienza Renale Cronica, Iperparatiroidismo secondario, Attivatori dei VDR, Paracalcitolo

Dichiarazione di conflitto di interesse: Gli Autori dichiarano di non avere conflitto di interessi.

Indirizzo degli Autori:

Dr.ssa Marina Di Luca

Via dell'Abbondanza 29

61121 Pesaro

m.diluca@ospedalesansalvatore.it

\section{Bibliografia}

1. Levin A, Bakris GL, Molitch M, et al. Prevalence of abnormal serum vitamin D, PTH, calcium, and phosphorus in patients with chronic kidney disease: Results of the study to evaluate early kidney disease. Kidney Int 2007; 71: 31-8.

2. Dusso A, Gonzales EA, Martin KJ, et al. Vitamin D in chronic kidney disease. Best Practice \& Research Clinical Endocrinology \& Metabolism 2011; 25: 647-55.

3. Cunningham J, Zhender D. New Vitamin D analogs and changing therapeutic Paradigms. Kidney Int 2011; 79: 702-7.

4. Bover J, Cozzolino M. Mineral and bone disorders in chronic kidney disease and end-stage renal disease patients: new insights into vitamin D receptor activation. Kidney Int Suppl 2011; 1: 122-9.

5. Palmer SC, McGregor DO, Craig JC. Vitamin D compounds for people with chronic kidney disease not requiring dialysis. Cochrane Database Syst Rev 2009; (4): CD008175.

6. Coburn JW, Maung HM, Elangovan L, et al. Doxercalciferol safely suppresses PTH levels in patients with secondary hyperparathyroidism associated with chronic kidney disease stages 3 and 4. Am J Kidney Dis 2004; 43: 877-90.

7. Cheng J, Zhang W, Zhang X, et al. Efficacy and safety of paricalcitol therapy for chronic kidney disease: a meta-analysis. Clin J Am Soc Nephrol 2012; 7 (3): 391-400.

8. Han T, Rong G, Quan D, et al. Meta-analysis: the efficacy and safety of paricalcitol for the treatment of secondary hyperparathyroidism and proteinuria in chronic kidney disease. Biomed Res Int 2013.

9. Coyne D, Acharya M, Qiu P, et al. Paricalcitol Capsule for the Treatment of Secondary Hyperparathyroidism in Stages 3 and 4
CKD. Am J Kidney Dis 2006; 47 (2): 263-76.

10. Hervás Sánchez JG, Prados Garrido MD, Polo Moyano A, et al. Effectiveness of treatment with oral paricalcitol in patients with pre-dialysis chronic kidney disease. Nefrologia 2011; 31 (6): 697-706.

11. Cozzolino M, Bruschetta E, Stucchi A, et al. Role of Vitamin D Receptor Activators in Cardio-Renal Syndromes. Sem Nephr 2012; 32 (1): 63-6.

12. Chen S, Law CS, Grigsby CL, et al. Cardiomyocyte-specific deletion of the vitamin $\mathrm{D}$ receptor gene results in cardiac hypertrophy. Circulation 2011; 124: 1838-47.

13. Bodyak N, Ayus JC, Achinger S, et al. Activated vitamin D attenuates left ventricular abnormalities induced by dietary sodium in Dahl salt-sensitive animals. Proc Natl Acad Sci U S A 2007; 104 (43): 16810-5.

14. Park WC, Oh YS, Shin YS, et al. Intravenous calcitriol regresses myocardial hyperthrophy in hemodialysis patients with secondary hyperparathyroidism. Am J Kidney Dis 1999; 33: 73-81.

15. Thadhani R, Appelbaum E, Pritchett Y, et al. Vitamin D Therapy and Cardiac Structure and Function in Patients With Chronic Kidney Disease. The PRIMO Randomized Controlled Trial. JAMA 2012; 307 (7): 674-84.

16. Fedele F, Frati G, Biondi-Zoccai G. Questioning the validity of a recent randomized trial on paricalcitol in patients with echocardiographic evidence of cardiac hypertrophy. Int J Cardiol 2012.

17. Tamez H, Zoccali C, Packham D, et al. Vitamin D reduces left atrial volume in patients with left ventricular hypertrophy and chronic kidney disease. Am Heart J 2012; 164: 902-9.

18. Ristow B, Ali S, Whooley MA, Schiller NB. Usefulness of left atrial volume index to predict heart failure hospitalization and 
mortality in ambulatory patients with coronary heart disease and comparison to left ventricular ejection fraction (from the Heart and Soul Study). Am J Cardiol 2008; 102: 70-6.

19. Dusso A. Defective renal maintenance of the vitamin D endocrine system impairs vitamin D renoprotection: a downward spiral in kidney disease. Kidney Int 2011; 79: 715-29.

20. Li YC. Renoprotective effects of vitamin D analogs. Kidney Int 2010; 78: 134-9.

21. Eijkelkamp WB, Zhang Z, Remuzzi G, et al. Albuminuria is a target for renoprotective therapy independent from blood pressure in patients with type 2 diabetic nephropathy: post hoc analysis from the Reduction of Endpoints in NIDDM with the Angiotensin II AntagonistLosartan (RENAAL) trial. J Am Soc Nephrol 2007; 18: 1540-6.

22. Agarwal R, Acharya M, Tian J, et al. Antiproteinuric effect of oral paricalcitol in chronic kidney disease. Kidney Int 2005; 68: 2823-8.

23. Alborzi P, Patel NA, Peterson C, et al. Paricalcitol reduces albuminuria andinflammation in chronic kidney disease: A randomized doubleblind pilot trial. Hypertension 2008; 52: 211-2.

24. Fishbane S, Chittineni H, Packman M, et al. Oral paricalcitol in the treatment of patients with CKD and proteinuria: a randomized trial. Am J Kidney Dis 2009; 54: 647-52.
25. de Zeeuw D, Agarwal R, Amdahl M, et al. Selective vitamin D receptor activation with paricalcitol for reduction of albuminuria in patients with type 2 diabetes (VITAL study): a randomised controlled trial. Lancet 2010; 376: 1543-51.

26. De Nicola, Conte G, Russo D, et al. Antiproteinuric effect of add-on paricalcitol in CKD patients under maximal tolerated inhibition of reninangiotensin system: a prospective observational study. BMC Nephrol 2012; 13: 150.

27. Liu LJ, Lv JC, Shi SF, Chen YQ, Zhang H, Wang HY. Oral Calcitriol for Reduction of Proteinuria in Patients With IgA Nephropathy: A Randomized Controlled Trial. Am J Kidney Dis 2012; 59 (1): 67-74.

28. Jørgensen HS, Winther S, Povlsen JV, Ivarsen P. Effect of vitamin-D analogue onalbuminuria in patients with non-dialysed chronic kidney disease stage 4-5: a retrospective single center study. BMC Nephrol 2012; 13: 102.

29. Shoben A, Rudser KD, de Boer IH, et al. Association of Oral Calcitriol with Improved Survival in Nondialyzed CKD. J Am Soc Nephrol 2008; 19: 1613-9.

30. Kovesdy CP, Ahmadzadeh S, Anderson JE, et al. Secondary hyperparathyroidism is associated with higher mortality in men with moderate to severe chronic kidney disease. Kidney Int 2008; 73: 1296-302. 